\title{
Distribuição de Direitos de Acesso e Licenças para o Gerenciamento de Conteúdos Digitais
}

Cleber V. Filippin ', Valério Rosset ${ }^{1}$, Carla M. Westphall ${ }^{2}$

Faculdades Integradas da Rede de Ensino Univest - FACVEST

${ }^{2}$ Universidade Federal de Santa Catarina - UFSC

nnemesis, valerio, carla\}@lrg.ufsc.br

Abstract. This article proposes an architecture for distributing and managing the digital contents, combining traditional access control and Digital Rights Management (DRM) aspects, which executes the content usage control. The architecture defines security policies to establish access rights to protected content and to establish licenses to control the use of digital contents at the client side. This work also defines models for the authorization and rights distribution entity and for the license creation, distribution and managemen entity. Prototypes are implemented using XML-based technologies, which facilitates the integration of different modules of the architecture.

Resumo. Este artigo propõe uma arquitetura de distribuição de direitos de acesso e licencas para o gerenciamento de conteudos digitais, combinando aspectos de controle de acesso tradicional e Digital Rights Managemen (DRM), que executa o controle de uso do conteúdo. A arquitetura define politicas de segurança para estabelecer direitos de acesso a conteído protegido e para estabelecer licenças para controlar o uso de conteúdo. digitais no lado cliente. Este trabalho também define modelos para a entidade de autorização e distribuição de direitos e para a entidade de criacão, distribuição e gerenciamento de licenças. Protótipos são implementados usando tecnologias baseadas em XML, que facilitam a integração dos diferentes módulos da arquitetura.

\section{Introducão}

O crescimento da Internet como meio de distribuição e a evolução das tecnologias digitais possibilita o surgimento de novos modelos e oportunidades de negócios para distribuição de conteúdos na forma digital. Entretanto, as formas tradicionais de controle de direitos sobre conteúdos como leis, contratos e criptografia, não são suficientemente eficientes para impedir ou diminuir o problema da distribuição ilegal de conteúdos digitais, trazendo a necessidade de desenvolvimento de modelos e tecnologias para gerenciamento, segurança, controle e automatização do fluxo de conteúdo e do acesso a serviços na Internet [Park et. al 2000]

O nível de proteção desejado vai além da simples segurança para a distribuicão do conteúdo digital; é necessário que existam maneiras de fornecer uma proteção persistente do conteúdo, ou seja, proteção que permanece com o conteúdo depois deste ser transmitido [Erickson et al. 2001]. Digital Rights Management (DRM) é o termo comum associado com tecnologias capazes de proteger conteúdos digitais após distribuição, proporcionando maneiras de exercer controle de uso sobre o conteúdo. Nos trabalhos existentes na literatura [Rosenblatt et al. 2002]. [Chong et al. 2002]. [Park e 
al. 2000] e [Park and Sandhu 2002], existem propostas de alguns modelos para gerenciamento e controle do acesso e do uso dos conteúdos digitais. Entretanto, ainda existem várias questões sem solução, como a questão da interação entre o controle de acesso tradicional dos conteúdos digitais e o controle de uso, provida pelos modelos DRM. Nesse contexto, este artigo propõe uma arquitetura para distribuição de direitos e licenças para o gerenciamento de conteúdos digitais, unindo aspectos do controle de acesso tradicional e aspectos de DRM que realizam o controle de uso do conteúdo. A arquitetura trata os aspectos da autorização de acesso aos conteúdos, defínindo a interação entre os componentes do controle de acesso e os componentes de controle de uso. Trata também da criação, da distribuição e do gerenciamento de licenças de uso dos conteúdos digitais

$\mathrm{O}$ artigo está organizado da seguinte forma: a seção 2 apresenta uma visão geral sobre DRM e os trabalhos de pesquisa relacionados. A arquitetura para distribuição e gerenciamento de conteúdos digitais é apresentada na seção 3, definindo aspectos da autorização, da criação, distribuição e gerenciamento de licenças. Os protótipos desenvolvidos também são descritos na seção 3. Conclusões são apresentadas na seção 4.

\section{Digital Rights Management (DRM)}

DRM envolve a descrição, identificação, comercialização, proteção, monitoramento e rastreamento de formas de direitos de uso sobre conteúdos, incluindo o gerenciamento de relacionamentos entre detentores de direitos [lanella 2001]. Soluções que possibilitam proteção persistente podem incluir componentes tecnológicos como empacotadores de conteúdo, controladores, servidores de licenças, rastreamento de acesso ou de uso de conteúdo, e licenciamento de direitos [Rosenblatt et al. 2002]. Sistemas DRM fornecem proteção persistente e gerenciam o conteúdo, baseados em regras de uso especificadas pelo proprietário ou distribuidor do conteúdo, e nos direitos detidos pelo usuário.

Atualmente, existem diversas tecnologias e soluções criadas para atenderem objetivos e modelos de negócios no mercado de DRM. Tentativas de padronização incluem a linguagem de especificação de direitos XrML (eXtensible rights Markup Language) e o protocolo ICE (Interchange Content Exchange) [Rosenblatt et al. 2002]. Soluções proprietárias incluem soluções específicas para determinados formatos e modelos de negócios, e soluções mais abrangentes, que podem ser utilizadas com vários formatos e modelos de negócios. Mas soluções proprietárias são geralmente fechadas, e não especificam quais tecnologias são usadas, nem como são realizadas as tarefas de proteção de conteúdo e controle de uso, ou seja, não existem padrões que estabeleçam uma maneira especifica para disseminar e controlar o acesso sobre conteúdos digitais.

\subsection{Trabalhos de Pesquisa Relacionados}

Trabalhos de pesquisa envolvem modelos e arquiteturas DRM, mas não são determinantes e esclarecer técnicas para implementar o controle sobre conteúdos digitais. Em [Chong et al. 2002] é apresentado o projeto SUMMER (Seclire MultiMedia Retrieval), que envolve apenas uma arquitetura fim-a-fim para gerenciamento de bases de dados multimidia, envolvendo duas partes distintas, uma para identificacão, autenticação, autorização; outra parte para executar funçoes de servidor de licenças. Em [Rosenblatt et al. 2002] é proposta uma arquitetura de referência para soluções DRM, composta por três elementos principais: o servidor de (achas e o cliente. LPark et al. 20001 anal cona taxonomia de arquiteturas de controlada de informação digital apresentando controle eguranca baseadas

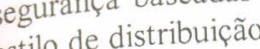

No trabalho de pesquisa em [Park and Sandhu 2002], a noção de controle de uso

(Originator (Usage Control - UCON) e apresentado e unido baseado em políticas.

Control), um mecanismo de controle de acesso base percebe a necessidade de pesquisas Nos trabalhos de pesquisa analisados, percebe-se a nistemas DRM. Existe a mais aprofundadas em relação a aspectos como autorizaco e so suscando soluçes para necessidade de análise de mecanismos e soluções, e de estudos buscan e arquiteturas que problemas como o controle e gerenciamento de licença de acesso e de uso não são permitam implementar na prática os aspectos de encontrados nos trabalhos de pesquisa analisados.

\section{(a) Gerenciamento e Distribuição de Conteúdos Digitais} A arquitetura para gerenciamento e distribuição de conteúdos digitais (Digital Content Distribution and Managemes An apresenta uma divisão e distribuição dos é especializadas. A arquitetura (Figura 1) é formada pelas enticades Entity- AE); Entidade Cliente (Client); Entidade de Autenticação (Authentication Entity - AE); Entidade de Conteúdo (Content Entity - CE); Entidade de Criação, Distribuição e Ge Licenças (License Creation, Distribution and Management Entity Gerenciamento de Licenças (Authorization and Rights Distribution Entity - ARDE). A Entić é definida como uma aplicação de controle de pré é executada no lado cliente como um plugin.

Entidade de Autenticaco (AE) é responsável por recolher as informações para de Autenticação (AE) é responsável por redencial do usuário para necessárias para autenticar um usuário, e disponibilizar uma credencial do usuánio par de os outros componentes do sistema, funcionando como uma base de informaçães distribuir atributos de usuários. A Entidade de Conteúdo (CE) è responsavel por distribuir conteúdos protegidos aos usuários autenticados no sistema, e serve também como base de informacões de atributos de conteúdos protegidos e de informações relacionadas a pagamento e assinaturas.

A Entidade de Criacũo Distribuição e Gerenciamento de Licenças (LCDME) é responsável por criar e distribuir licenças usadas para o controle de uso de conteúdos protegidos, e o gerenciamento de uso destas licenças. O processo de geração de licenças é fires que usuário possui sobre os conteúdos digitais pre controle protegidos. Esses direitos são determinados através da avalição do pocão e Distribuição de acesso durante o processo de autorização. A Entiada de Aunorização e Distribuisũo de Direitos de acesso (ARDE) é responsável por avaliar politicas de controle de acesso de determinar quais os direitos que o usuário possui sobr

específico, e disponibilizá-los ao servidor de licenças.

A interação entre as entidades pertencentes à arquitetura DCDMA oconecificado de trocas de mensagens seguras. Os usuários exercem os direitos de acesso especificados por licenças, através de ações requisitadas a a

visualizaço ou execução de conteudos digitais. 


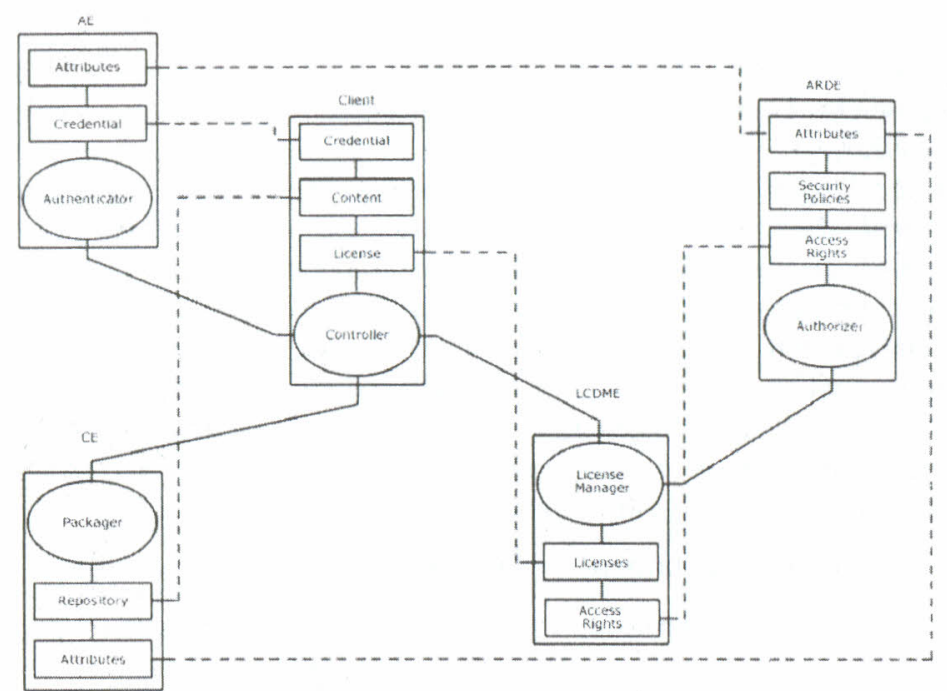

Figura 1- Arquitetura DCDMA

Este artigo apresenta a definição das entidades ARDE e LCDME, e aborda suas estruturas internas, comunicação e implementação dos protótipos.

\subsection{Modelo da Entidade de Autorização e Distribuição de Direitos de Acesso}

A entidade de autorização e distribuição de direitos é subdividida em componentes responsáveis por disponibilizar as políticas de segurança e atributos necessários para o processo de decisão, bem como componentes diretamente responsáveis pelo processo de decisão.

A definição de entidades envolvidas no processo de autorização como suporte a efetivação da arquitetura proposta é baseada no RFC-2904 AAA Authorization Framework da IETF [Vollbrecht 2000], que determina a distribuição de componentes em uma arquitetura de autorização genérica. A utilização desses componentes (individualmente ou em conjunto) de maneira distribuída representando as entidades da arquitetura proposta permite a não sobrecarga em uma entidade específica. O $A A A$ Authorization Framework da IETF define os seguintes componentes: o PEP (Policy Enforcement Point) é o componente responsável por requisitar e aplicar decisões dé acesso sobre algum recurso; o PDP (Policy Decision Point) é o componente responsável por avaliar requisições de acesso através de políticas de segurança e determinar se a requisição de acesso a um recurso é válida ou não; o PIP (Policy Information Point) é responsável por fornecer ao PDP valores de atributos de usuários, dos recursos e do ambiente; o PAP (Policy Administration Point) é responsável por criar e armazenar políticas de segurança; e o PRP (Policy Retrieval Point) é responsável por fornecer as politicas de segurança ao PDP.

O AAA Authorization Framework da IETF é bastante flexível e permite implementar a autorização de forma distribuida e independente, sendo que a interação e organização entre os vários pontos são de responsabilidade do desenvolvedor. Esse framework foi utilizado também como base no modelo da linguagem para definição de políticas de segurança designada XACML (eXtensible Access Control Markup
Language) [Godik 2003]

A é implementada atraves de uma aplicação de

Na DCDMA, a entidade ARDE é implem esul em sua estru atorização e distribuição de direitos de acesso (ARDA), que possuido PDP, e PRP. Da autoriza componentes que realizam as mesmas funcionalidades do PDP e conce de PAP é mesma maneira a entidade LCDME pode ser vista como o PEP. Os entidades, devido mesmádo nesta arquitetura. Já o PIP pode estar presente em todas as elas.

abstraido necessidade de troca de informações sobre atributos entre elas.

ao fato da neces dos padrões baseados em XML, que são aplicados para estabelecer a geração e troca de informaçoes seguras entre flexibilidade e interoperabilidade não acrescenta ao modelo DCDMA caracteristicas de fexses padrões não fazem parte da encontradas em outras arquiteturas. Efetivan tornar desnecessária a criação de protocolos arquitetura, mas o uso desses padrōes pode tornar desneces
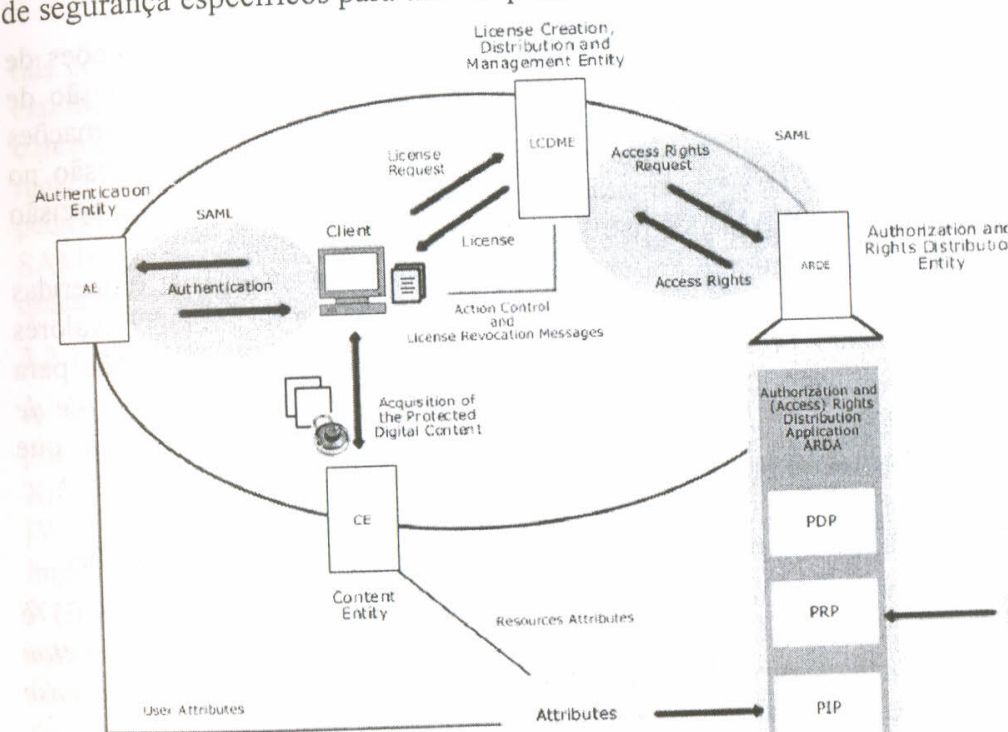

Figura 2 - DCDMA, ARDE e Padrões de segurança XML.

As trocas de menagens de autorizaço e distribuição de direitos de acesso são de segurança SAML (Secure Assertion implementadas através do uso do padrão de segurança SAM Markup Language) [Maler 2003]. As politicas de segurança São impl Markup Language) do uso do padrão de segurança XACML (eXtensible Access Control Mankup Langude [Godik 2003]. É visível que apenas SAML e XACML não garantem a integridade autenticidade de mensagens, apenas fornecem vocabulários para estabelecer requisiçoes e respostas de acesso, autenticação, atributos e definição de políticas respectivamente. Assim, para reforçar a segurança na troca de mensagens entre as entidades pode-se utilizar outros padrões de segurança XML.

\subsubsection{Implementação da Entidade ARDE}

O protótipo foi desenvolvido como um framework para aplicações Java, e contém as diversas classes que representam os diferentes módulos da estrutura para uma aplicação 
ARDA. A implementação dessas classes permite que outras aplicações possam utilizá-las de maneira individual. Assim, desenvolvedores podem utilizar as classes de maneira a implementar a melhor solução para suas necessidades. Os pacotes utilizados para desenvolvimento foram o OpenSAML [Opensaml 2002] versão 1.0, SunXACML [Sunxacml 2003] e o parser Xerces.

O protótipo implementado determina como será o acesso a todos os conteúdos que estarão sob domínio dos usuários. O processo de autorização é executado por uma aplicação de middleware. Uma aplicação de autorização e distribuição de direitos de acesso (ARDA) é dividida em diferentes módulos que executam as funções referentes ao PDP, PRP e PIP, além de outros módulos responsáveis por receber, enviar e formatar mensagens. A estrutura da aplicação ARDA é formada pelos módulos Listen/Response, Encapsulamento, Desencapsulamento, Recuperação de Politicas, Recuperação de Atributos e Controle de Decisão.

O módulo Listen/Response é responsável pelo recebimento de requisições de autorização sobre direitos e por enviar as mensagens de resposta sobre a decisão de autorização. O módulo de Encapsulamento é responsável por coletar as informações contidas nas requisições SAML que chegam e repassá-las ao controle de decisão no formato desejado. O módulo Desencapsulamento é responsável por formatar a decisão de autorização em um padrão SAML de mensagem de resposta desejada.

O módulo de Recuperacão de Politicas agrupa as políticas necessárias requeridas pelo controle de decisão. O módulo de Recuperação de Atributos disponibiliza valores de atributos, que não estão presentes na requisição de autorização, necessários para recuperação de políticas envolvidas no processo de decisão. Finalmente o Controle de Decisão efetua a avaliação de políticas através do uso de algoritmos de avaliação, que geram a decisão de autorização sobre os direitos.

\subsection{Modelo da Entidade de Criação, Distribuição e Gerenciamento de Licenças}

A Entidade de Criação, Distribuicão e Gerenciamento de Licencas (LCDME) constituída pelos módulos Rights Interpretation Module (RIM), License Creation Module (LCM), License Distribution and Storage Module (LDSM), e License Management Module (LMM), conforme Figura 3.

O módulo RIM recebe a mensagem com a decisão de direitos, provinda da ARDE, interpreta a mensagem em formato SAML, extraindo os direitos contidos na mensagem e colocando-os em uma lista, enviada ao módulo LCM para criação de licenças. O módulo RIM também formata e envia as mensagens de requisição de direito para a ARDE. O módulo LCM usa a linguagem XrML para criar as licenças digitais, com base na lista de direitos emitida pelo RIM. As informações de identificação do principal são enviadas pelo controlador no lado cliente, assim como as informacões sobre o conteúdo. A licença é armazenada em um arquivo XML, e enviada ao módulo LDSM. No LCM, são implementados um processo interpretador de licenças e um processo de validação de condições, para verificar a integridade das licenças criadas. Estes processos de interpretação e validação também são implementados no controlador cliente e no LMM (para verificação de datas de expiração). O módulo LDSM executa as tarefas de distribuição da licença para o cliente, e armazenamento da licença numa base de dados $\mathrm{XML}$, para controle e gerenciamento. As licenças são enviadas para o controlador no cliente, e o mesmo as armazena de forma segura na máquina cliente, e executa o gerenciamento de uso. O controlador envia mensagens periódicas para o LMM, com informações sobre o uso da licença.

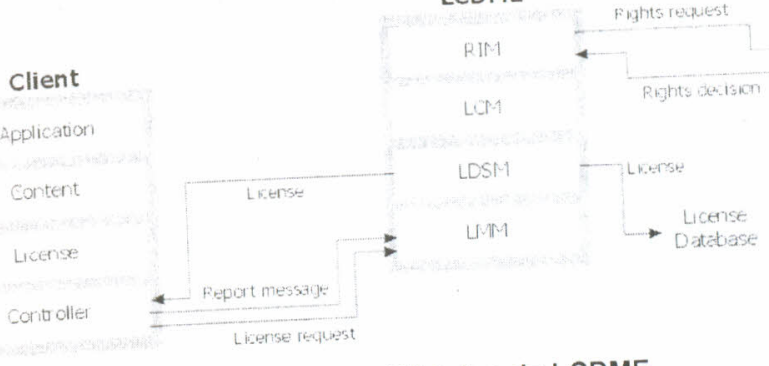

Figura 3. Módulos da LCDME.

de licenças no lado servidor O módulo LMM executa tarefas de gerenciansens avisando ao cliente sobre a tais como verificar datas de expiração e enviar mensagens enviados pela aplicação expiração da licença, além de verificar, a partir dos relatónios enido. O LMM também expirça se uma licença está sendo usada de modo indevido. O LMM também controladora, Entidade Cliente (a partir do controlador), envia os recebe os pedidos de licença da Encição em formato pedidos para o RIM, que faz a formataça do dados XML são excluídas pedicencas armazenadas na base de dados quando expiram.

3.2. Implementação da Entidade LCDME

A implementação da LCDME é realizada com o uso da linguagem C++ para é utilizada a linguagem de especificação de direitos programação dos módulos. Também é utilizada a linguagem (Software Development Kit) XrML para a criação de licenças, atraves do XIM [Wang et al. 2002], fornecido pela ContentGuard, empresa ciadora en linguagem XrMt. A linguagem XrML for escolhida por ser XrML firios desenvolvedores de XML e por ser bem aceita no mercado, sendo utiliza foi MSXML 4.0, da Microsoft aplicações DRM, como a Microsoft. O parser utilzado for MSX̃es foram desenvolvidos Os processos de interpretação de licenças e validação de condicones fó com base nos exemplos do XrML SDK. O parser XML usado no modulo LCDME Slatform SDK,

MSXML 4.0, da Microsoft. Também é configurado o Mic
necessário para desenvolvimento de aplicações para Windows.

\section{Conclusões}

Este artigo propôs uma arquitetura para distribuição de direitos de acesso digitais, dradicional e aspectos de DRM que realizam o unindo aspectis

controle de uso do conteúdo.

arquitetura proposta políticas de segurança para estabelecer RDE que pode utilizar politicas de uma linguagem padrão de direitos de acesso ao conteúdo protegido, atraves do uso de uma linguagem padro de definição de política, a XACML. Quanto ao controle de uso, a arquitetura licenças digitais usou ainda outra tecnologia baseada em XML para especificação de licenças digitais (XrML), que possibilita o controle de uso depois da distribuição do conteúdo. Diferente dos trabalhos analisados na literatura, a arquitetura proposta proporciona o controle

uso de licenças também no lado do servidor dificultando o uso inadequado das licenças.

A DCDMA promoveu a interação entre os componentes do co 
os componentes de controle de uso utilizando padrão SAML e incorporando aspectos de segurança que são inexistentes na literatura relacionada. O desenvolvimento do protótipo contribuiu para refinar alguns aspectos importantes da arquitetura, atuando diretamente na interligação entre o controle de acesso tradicional e o controle de uso. A definição de padrões de segurança e comunicação específicos para DRM, como trabalhos futuros, é de grande importância, na medida que sistemas operacionais modernos tendem a implementar esse tipo de controle de acesso.

\section{Referências Bibliográficas}

Chong, J.C.N., Buuren, R.V., Hartel, P.H. and Kleinhuis, G. (2002) "Security Attributes Based Digital Rights Management", In: Joint International Workshop on Interactive Distributed Multimedia Systems/Protocols for Multimedia Systems (IDMS/PROMS), Portugal, November, $18 \mathrm{p}$.

Erickson, J.S., Williamson, M., Reynolds, D., Vora, P. and Rodgers, P. (2001) "Principles for Standardization and Interoperability in Web-based Digital Rights Management", In: W3C Workshop on Digital Rights Management, France, January.

Godik, S. and Moses T. "eXtensible Access Control Markup Language (XACML) Version 1.0". OASIS Standard, 18 February 2003.

Ianella, R. (2001) "Open Digital Rights Management", In: W3C Workshop on Digital Rights Management, France, January, 5 p.

Maler, E., Mishra P., Philpott, R. "Assertions and Protocol for the OASIS Security Assertion Markup Language (SAML) V1.1”, OASIS Standard, 18 July 2003.

Park, J., Sandhu, R. and Schifalacqua, J. (2000) "Security Architectures for Controlled Digital Information Dissemination", In: 16 $6^{\text {th }}$ Annual Computer Security Application Conference, USA, December, p. 224-233.

Park, J. and Sandhu, R. (2002) "Originator Control in Usage Control", In: $3^{\text {rd }}$ International Workshop on Policies for Distributed Systems and Networks, USA, June, p. 60-66.

Rosenblatt, B., Trippe, B. and Mooney, S. (2002) "Digital Rights Management Business and Technology", M\&T Books, $1^{\text {st }}$ Edition, USA, $288 \mathrm{p}$.

Sun Microsystems, Inc. "Sun's XACML Implementation". 2003-2004. Disponivel em http://sunxacml.sourceforge.net/javadoc/index.html .

The Ohio State University Corporation for Advanced Internet Development. "The OpenSAML version 1". 2002. Disponível em

http://wayf.internet2.edu/opensaml/java/doc/api/index.html.

Vollbrecht, J. et al. "AAA Authorization Framework". The Internet Society, RFC-2904, August 2000.

Wang, X., Lao, G., DeMartini, T., Reddy, H., Nguyen, M. and Valenzuela, E. (2002) "XrML - eXtensible rights Markup Language", In: ACM Workshop on XML Security, USA, November, p. 71-79. 